\title{
On the mechanism of charge transport in pentacene
}

\author{
H. A. v. Laarhoven, ${ }^{1, a)}$ C. F. J. Flipse, ${ }^{1}$ M. Koeberg, ${ }^{2}$ M. Bonn, ${ }^{2}$ E. Hendry, ${ }^{3}$ G. Orlandi, ${ }^{4}$ \\ O. D. Jurchescu, ${ }^{5}$ T. T. M. Palstra, ${ }^{5}$ and A. Troisi ${ }^{6}$ \\ ${ }^{1}$ Department of Applied Physics, Eindhoven University of Technology, P.O. Box 513, 5600 MB Eindhoven, \\ The Netherlands \\ ${ }^{2}$ FOM Institute for Atomic and Molecular Physics, Kruislaan 407, 1098 SJ Amsterdam, \\ The Netherlands \\ ${ }^{3}$ School of Physics, University of Exeter, Stocker Road, Exeter EX4 4QL, United Kingdom \\ ${ }^{4}$ Dipartimento di Chimica, Università di Bologna, Via F. Selmi 2, 40126 Bologna, Italy \\ ${ }^{5}$ Zernike Institute for Advanced Materials, University of Groningen, Nijenborgh 4, 9747 AG Groningen, \\ The Netherlands \\ ${ }^{6}$ Department of Chemistry and Centre of Scientific Computing, University of Warwick, Coventry CV4 7AL, \\ United Kingdom
}

(Received 24 March 2008; accepted 10 June 2008; published online 23 July 2008)

\begin{abstract}
Terahertz transient conductivity measurements are performed on pentacene single crystals, which directly demonstrate a strong coupling of charge carriers to low frequency molecular motions with energies centered around $1.1 \mathrm{THz}$. We present evidence that the strong coupling to low frequency motions is the factor limiting the conductivity in these organic semiconductors. Our observations explain the apparent paradox of the "bandlike" temperature dependence of the conductivity beyond the validity limit of the band model. (ㅇ 2008 American Institute of Physics.
\end{abstract}

[DOI: $10.1063 / 1.2955462$ ]

Recent years have seen much interest in the conductivity of pentacene and other molecular semiconductors of the oligoacene family, fueled by the unique properties of these materials. Oligoacenes are flexible, cheap, and exhibit relatively high charge carrier mobilities, ${ }^{1}$ allowing for novel applications in electronic devices. ${ }^{2-4}$ Despite this interest, the nature of charge transport in the materials has remained the subject of intense debate.

Crystalline organic semiconductors exhibit charge mobilities that decrease with temperature.$^{5-9}$ In analogy to inorganic semiconductors, this has been interpreted as an indication of delocalized charge carriers and a "bandlike" transport mechanism. ${ }^{10-13}$ Theoretical models based on polaronic band conduction ${ }^{14}$ indeed provide a good qualitative description of the bandlike mobility of charge carriers in pentacene, but the factors determining the absolute charge mobility remain unknown. For instance, an analysis of the high temperature mobility has indicated that the mean free path of charge carriers is of the same magnitude as the intermolecular distance. ${ }^{15}$ It is not clear as to why the signature of delocalized bandlike transport persists at these temperatures, where the mean free path is smaller than the intermolecular distances. Because of the "softness" of these van der Waals bonded organic crystals, it is possible that the coupling of the carriers with the intermolecular vibrations could play a key role in the description of the charge carrier dynamics. Troisi and Orlandi ${ }^{16}$ have recently formulated a model (TO model) that states that the charge mobility in oligoacenes is limited by large fluctuations of the electronic coupling between adjacent organic molecules due to thermal molecular motions, in particular, the low energetic phonon modes with energies

${ }^{a)}$ Electronic mail: rik1978@gmail.com. around $1 \mathrm{THz} .{ }^{17}$ This model correctly describes the bandlike temperature dependence of the mobility, including its absolute value, outside the validity of delocalized transport models. ${ }^{16}$ Direct experimental evidence for the various proposed models has been lacking. In particular, experimental insights into the role of intermolecular low frequency vibrational modes in determining the charge transport mechanism have been missing to date.

Terahertz spectroscopy ${ }^{18}$ provides the opportunity of studying the response of charge carriers in the frequency range of these modes, and has proven useful for investigating photocarrier dynamics in several organic semiconducting materials. ${ }^{5-7,19-21}$ By recording the modulation of the transmission of terahertz probe pulses after optically creating carriers in the sample, the technique allows for the determination of the photoconductivity spectrum in the range of $0.2-$ 1.2 THz. The terahertz conductivity spectrum has been shown to contain detailed information on the nature of the charge transport. Band-type ${ }^{22}$ and disorder-induced dispersive $^{19}$ transport, for example, have distinct spectral signatures.

We study ultrapure, vapor grown single crystals of pentacene with a trap density less than $2 \times 10^{17} \mathrm{~cm}^{-3}$ and typical dimensions of $5 \mathrm{~mm} \times 5 \mathrm{~mm} \times 20 \mu \mathrm{m} .{ }^{8}$ These crystals are placed in a $\mathrm{He}$ cryostat at a pressure below 1 $\times 10^{-3} \mathrm{mbar}$, to both control temperature and avoid effects of (photo-) oxidation. ${ }^{23}$ Mobile charges are generated in pentacene (absorption gap $1.85 \mathrm{eV}$ ) by laser excitation using 266 $\mathrm{nm}(4.7 \mathrm{eV})$ and $400 \mathrm{~nm}(3.1 \mathrm{eV})$ femtosecond laser pulses. The terahertz transient conductivity (terahertz-TC) is determined using terahertz spectroscopy, using the setup described in detail in Ref. 18. Briefly, terahertz probe pulses are created by optical rectification of $800 \mathrm{~nm}, 120$ fs laser pulses 

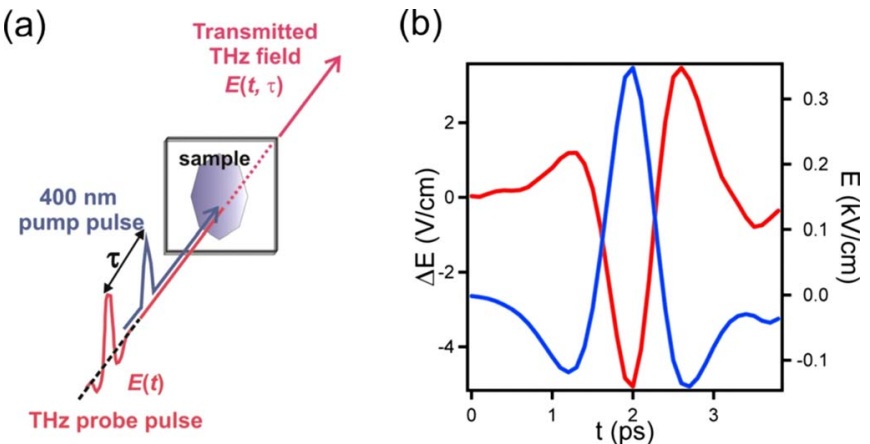

FIG. 1. (Color online) (a) Schematic representation of the experiment. The 266 or $400 \mathrm{~nm}$ laser pump pulses create photocarriers in the sample and at a time $\tau$ later the terahertz probe pulse $E(t)$ travels through the sample, resulting in a transmitted signal $E_{\text {trans }}(t, \tau)$. (b) Scans of the transmission $E_{\text {trans }}(t) \quad$ [blue (bottom) trace] and modulation $\Delta E_{\text {trans }}(t)=E_{\text {trans }}(t)$ $-E_{\text {trans }}\left(t, \tau^{\prime}\right)$ [red (top) trace]; average over 20 scans and $\tau^{\prime}=1.0$ ps. These measurements were performed at $20 \mathrm{~K}$ using $400 \mathrm{~nm}$ pump pulses to photoinject charge carriers.

in a ZnTe crystal, thereby creating single cycle oscillations of an electric field (peak field $\sim 1 \mathrm{kV} / \mathrm{cm}, \sim 1.5$ ps duration). The pulses probe conductivity in the a-b plane of the crystal under normal incidence. The transmitted field $E_{\text {trans }}(t)$ is measured in the time domain (with subpicosecond temporal resolution) by electro-optic sampling. The frequency dependent, complex conductivity $\sigma(\omega, \tau)$ at time $\tau$ after the generation of carriers by the pump pulse, is readily determined from the change in the transmitted terahertz pulses $\Delta E_{\text {trans }}(t, \tau)$ [see Fig. 1(a)], ${ }^{18}$

$$
\sigma(\omega, t)=\sigma^{\prime}(\omega, \tau)+i \sigma^{\prime \prime}(\omega, \tau) \propto \Delta E_{\text {trans }}(\omega, \tau) / E_{\text {trans }}(\omega),
$$

where the angular frequency $\omega=2 \pi / t$ is the Fourier conjugate of the time $t$.

The initial decay of the modulation after optically creating free charges is of the order of the terahertz pulse width. This means that care must be taken to extract the conductivity at short delay times. ${ }^{24} \mathrm{We}$ follow the procedure described in Ref. 24, which involves reading out the modulation of every point of the terahertz field at constant time after the photogeneration of charge carriers, i.e., $\Delta E_{\text {trans }}\left(t, \tau^{\prime}\right)$ for constant values of $\tau^{\prime}=\tau+t$. Typical shapes of transmission and modulation traces are shown in Fig. 1(b). We note that the signal levels in these experiments are such that the noise on $\Delta E_{\text {trans }}\left(\omega, \tau^{\prime}\right)$ and hence $\sigma\left(\omega, \tau^{\prime}\right)$ is very small compared to the signal.

Figure 2(a) shows the conductivities extracted using this procedure (for $400 \mathrm{~nm}$ excitation at $20 \mathrm{~K}$ ). The top trace is recorded $0.7 \mathrm{ps}$ after the maximum response. A marked frequency dependence of the complex conductivity is observed, most notably at early times: the traces of both the real and imaginary conductivities are indicative of a resonance at $1.1 \pm 0.1 \mathrm{THz}$. The real (dissipative) part peaks at this frequency, while the (derivativelike) imaginary part has the highest slope at this frequency. The resonance becomes less intense at longer delay times but is clearly visible up to 7.5 ps after excitation, and was observed in measurements on several samples [see Fig. 2(b)]. The resonance is observed

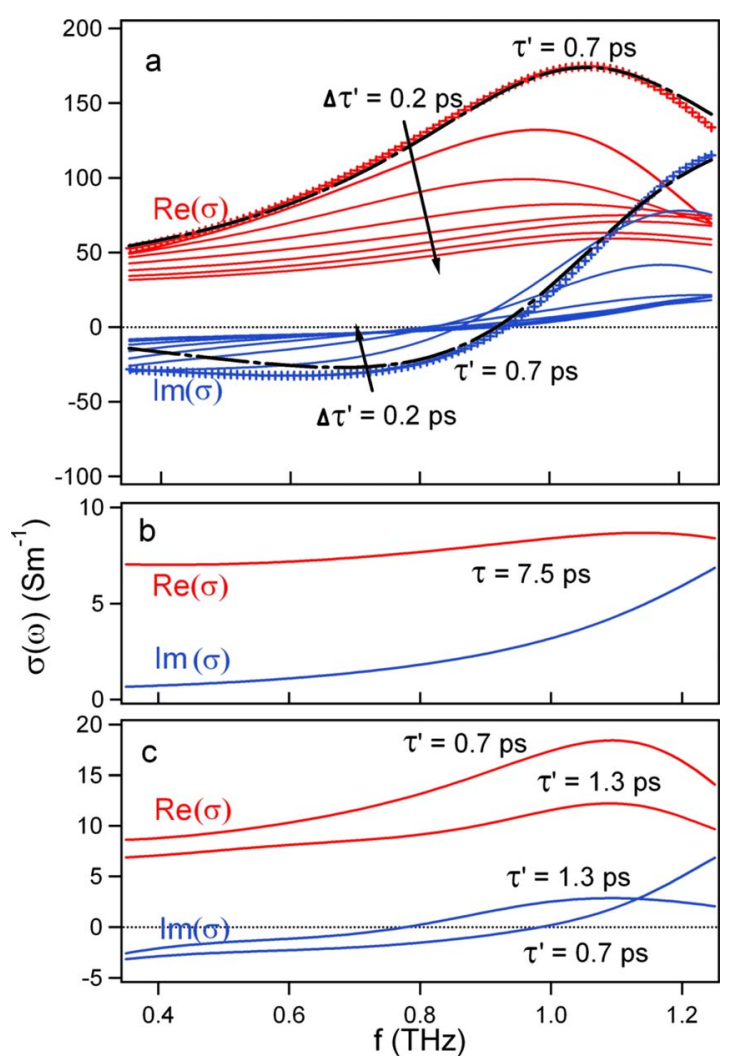

FIG. 2. (Color online) (a) Real (in red) and imaginary (in blue) components of the conductivity as a function of frequency at $20 \mathrm{~K}$ after exciting pentacene with $400 \mathrm{~nm}$ pulses with a laser fluence of $20 \mathrm{~J} \mathrm{~m}^{-2}$ (all traces represent an average of 20 scans). The spectra are shown for different times $\tau^{\prime}$ after excitation, ranging from 0.7 to $2.1 \mathrm{ps}$ (with intervals of $0.2 \mathrm{ps}$ ). For the conductivity at $\tau^{\prime}=7.5 \mathrm{ps}$, the original data points are shown. The dasheddotted line shows a fit function consisting of a frequency independent part (which represents the response from the free carriers) (Refs. 6 and 7) and a Lorentzian, showing that the conductivity data for $\tau^{\prime}=0.7$ ps contain a resonant absorption feature at $\sim 1.06 \mathrm{THz}$. (b) Real and imaginary components of the conductivity at $\tau^{\prime}=7.5 \mathrm{ps}$ [performed under the same conditions as Fig. 2(a) but on a different sample and averaged over 50 scans]. (c) Real and imaginary components of the conductivity at room temperature when exciting at $266 \mathrm{~nm}$ with a fluence of $16 \mathrm{~J} \mathrm{~m}^{-2}$ for $\tau^{\prime}=0.7$ and $\tau^{\prime}=1.3 \mathrm{ps}$, averaged over 30 scans.

independent of temperature and excitation wavelength. Figure 2(c) shows the conductivity at room temperature when exciting the sample with $266 \mathrm{~nm}$ laser pulses. Note that the traces in Fig. 2(c) look identical in shape to those of Fig. 2(a), exhibiting a resonance around $1.1 \mathrm{THz}$. The response in Fig. 2(c) is about nine times lower than that in Fig. 2(a) this is partly due to a lower excitation density at $266 \mathrm{~nm}$ (factor of 2) but mainly because the carriers are less mobile at room temperature. The temperature dependence of the terahertz conductivity is shown in the inset of Fig. 5, and is in good agreement with the previous reports. ${ }^{5-7,20}$ It has previously been interpreted as a sign of bandlike conductivity in pentacene. $^{5-8,20}$

We only consider data recorded after 0.7 ps following photoexcitation since determining the conductivity immediately after photoexcitation (when a "jump" in photoconductivity occurs) is not straightforward. ${ }^{24}$ In Ref. 25 , it has been shown for rubrene that optically active, ground state vibrational modes in the terahertz region can give rise to resonances in the conductivity spectrum. This cannot explain the 


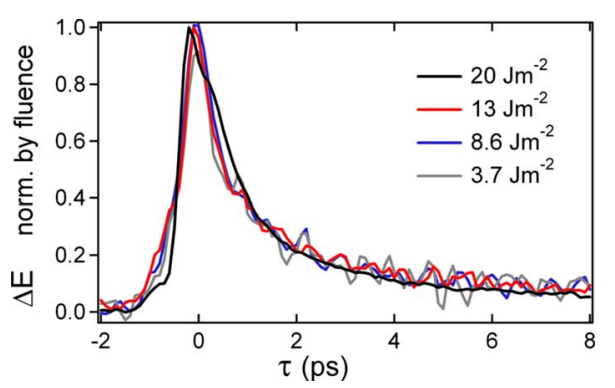

FIG. 3. (Color online) The spectrally integrated modulation $(\Delta E)$ as a function of delay time $\tau$ for pentacene optically excited at $400 \mathrm{~nm}(T=20 \mathrm{~K})$ with a laser fluence of 20 (black trace, averaged over 20 scans), 13 (red trace, 10 scans), 8.6 (blue trace, 10 scans) and $3.7 \mathrm{~J} \mathrm{~m}^{-2}$ (gray trace, 15 scans). The traces are normalized by their laser fluence.

resonances reported here: ground state absorption spectra measured in the frequency range of $0.3-1.3 \mathrm{THz}$ show a uniform, dispersionless response with an absorption less than $2 \%$ over the whole pentacene sample, indicating that the 1.1 $\mathrm{THz}$ resonance is not related to any ground state absorption of terahertz radiation. Numerical modeling of our data, following Ref. 26, reveals that the small shifts of the resonance frequency observed in the data (Fig. 2) can be explained by an excited state resonance at a fixed frequency of $1.1 \mathrm{THz}$.

Ai et al. ${ }^{21}$ recently observed a terahertz resonance upon photoexcitation of the polymer P3HT. They showed how several Drude-like models cannot account for this observation and attributed the resonance to bound polaron pairs, having observed a marked dependence of the dynamics on charge density. Since these polaron pairs only form at high carrier densities, the charge dynamics will change as a function of the laser fluence. In Fig. 3, the charge dynamics (the modulation $\Delta E$ as a function of delay time $\tau$ ) are depicted for pentacene $(T=20 \mathrm{~K})$ excited by $400 \mathrm{~nm}$ laser pulses with varying laser fluences. The traces have been normalized by their respective fluences. The fact that this normalization procedure results in four essentially indiscernible traces demonstrates that the dynamics do not depend on the charge density. Therefore, it seems safe to conclude that the resonance observed in this study is not due to polaron pairs.

Having ruled out ground state absorption and bipolaron effects, we conclude that the resonance at $1.1 \mathrm{THz}$ is analogous to the so-called soliton induced infrared (IR) absorption observed previously in conductive polymers such as polyacetylene at appreciably higher frequencies. ${ }^{27-29}$ As will be shown in the following, the $1.1 \mathrm{THz}$ resonance in pentacene corresponds to nuclear modes that strongly modulate intermolecular electron transfer, i.e., the modes with the largest off-diagonal electron phonon coupling. These are the same modes that limit the charge mobility according to the TO model. ${ }^{16}$ We justify this assignment by (i) the verification that the modes with the strongest off-resonance electronphonon coupling indeed have this frequency, and (ii) proving that upon charge injection these modes become strongly coupled to the oscillating electric field.

The frequency of the modes with the largest electron phonon coupling was computed following the procedure of Ref. 17 using a classical molecular dynamics simulation coupled to a series of quantum chemical computations. The

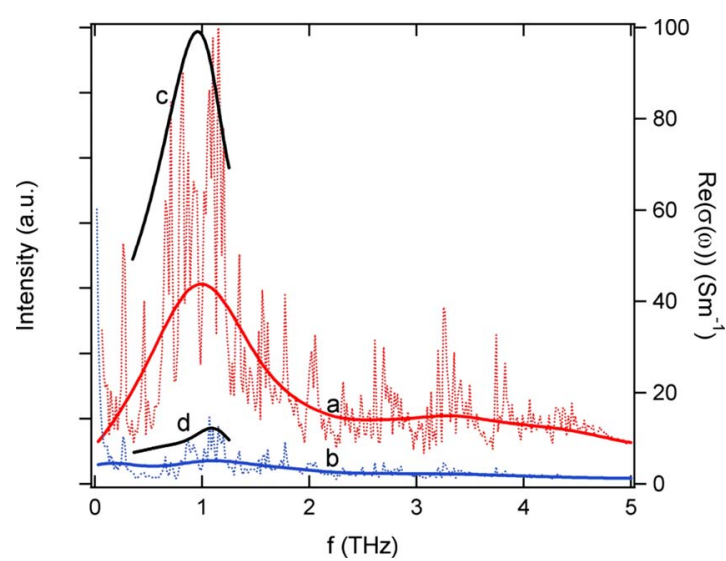

FIG. 4. (Color online) The Fourier transform of the autocorrelation function $\langle V(0) V(t)\rangle$ for both the coupling between the HOMO levels (a, in red) and LUMO levels (b, in blue). For simplicity, we show an average of the spectral density obtained for all the inequivalent pairs of nearest neighbors in the a-b plane since the difference between them is negligible when the broadening is introduced. The dotted traces show the original curves, whereas the solid ones represent the case of adding a broadening of $0.3 \mathrm{THz}$ to the original spectra. The black traces $c$ and $d$ show the conductivity (depicted on the right axis) at $t=1.3 \mathrm{ps}$ for $20 \mathrm{~K}$ when optically exciting at $400 \mathrm{~nm}$ (c) and at room temperature when optically exciting at $266 \mathrm{~nm}(\mathrm{~d})$.

time dependent transfer integral $V(t)$ between the highest occupied molecular orbitals (HOMOs) and the lowest unoccupied molecular orbitals (LUMOs) of neighboring molecules in the a-b plane of the pentacene crystal was evaluated to identify the frequency of the modes, which modulate most strongly the transfer integral. The Fourier transform of the autocorrelation function $\langle V(t) V(0)\rangle$ was calculated and the resulting spectral density is shown in Fig. 4. The spectral density varies little within the temperature range considered experimentally and among inequivalent pairs of the neighboring molecules. Clearly, the intermolecular transfer integral is modulated more strongly for the HOMO than for the LUMO and, more importantly, the modes that provide the strongest modulation are centered around $1 \mathrm{THz}$, i.e., very close to the resonance observed experimentally. This is even more clear when adding a small broadening of $10 \mathrm{~cm}^{-1}$ to the peaks in the spectrum to account for the temperature, possible inhomogeneities, and finite spectral resolution in the experiment (Fig. 4).

It is easy to qualitatively understand the strong carrierinduced coupling of these modes to the terahertz radiation. Any normal mode is coupled to the electromagnetic radiation if the charge density of the system is displaced when it is deformed along that mode, i.e., when the dipole derivative $\partial \mu / \partial q_{i} \neq 0$ ( $\mu$ is the dipole moment of the system). Since a displacement along nuclear modes with strong electronphonon coupling changes the charge carrier wave function dramatically, we expect these modes to be strong absorbers of radiation. For a more quantitative estimate, we consider the simple one-dimensional model Hamiltonian proposed in Ref. 16, describing the main physics of independent charges, whose dynamics are dominated by off-diagonal electron phonon coupling (note that this treatment requires separation, but not thermalization of electrons and holes), 


$$
\begin{aligned}
H= & \sum_{j}\left(-\tau+\alpha\left(u_{j+1}-u_{j}\right)\right)(|j\rangle\langle j+1|+| j+1\rangle\langle j|) \\
& +\sum_{j} \frac{1}{2} m \dot{u}_{j}^{2}+\sum_{j} \frac{1}{2} m \omega^{2} u_{j}^{2} .
\end{aligned}
$$

According to this model, localized on each molecule $j$, there is a single electronic state $|j\rangle$ and a harmonic nuclear mode $u_{j}$ with effective mass $m$ and frequency $\omega$ (i.e., these optical phonons are dispersionless). The nuclear displacements of molecules $j$ and its neighbor $j+1, u_{j}$, and $u_{j+1}$, respectively, modulate the transfer integral between $|j\rangle$ and $|j+1\rangle$ with the term $\alpha\left(u_{j+1}-u_{j}\right)$. The parameters of the Hamiltonian for pentacene $(\tau, \alpha, \omega)$ have been determined in Ref. 15 and they are valid at all temperatures. The radiation absorption rate at frequency $\omega$ is proportional to $M^{2}$ $=\sum_{i}\left|\partial \mu / \partial q_{i}\right|^{2}$ ( $i$ runs over the normal modes of the system), a quantity that can be easily computed from Eq. (2) at arbitrary temperature. Calculated values of the carrier-induced absorption are very high: For example, with parameters from Ref. 17 and the hole wave function delocalized over 10 molecules (a lower limit ${ }^{17}$ ) we find $M^{2}=17(\AA \mathrm{e})^{2}$.

The model of Eq. (2) can be integrated to describe the charge carrier dynamics at one particular energy. In the present experiment, the initial energy distribution of the carrier energy is not known and the model does not include the effect of thermal relaxation. However, the model provides two additional elements that will be used to interpret the experimental data: (i) the carrier mobility depends only weakly on its energy (the mobility of the highest energy state is about $20 \%$ larger than the mobility of the lowest energy state $)^{14}$ and (ii) the localization of the carrier is, instead, energy dependent, with the highest energy states having a localization length three to five times larger that the lowest energy state (the difference is larger at lower temperature). ${ }^{15}$ Considering that the absorption intensity depends on the fourth power of the localization length, the thermalization of the carriers could be monitored by the decrease in the absorption intensity of the modes at frequency $\omega$.

As stated above, the $1.1 \mathrm{THz}$ resonance is analogous to the soliton induced IR absorption observed previously in conductive polymers such as polyacetylene. ${ }^{27-29}$ In pentacene, as in polyacetilene, the modes that modulate the intersite coupling are crucial for the understanding of the charge transport mechanism. Our results demonstrate that these modes are in the terahertz frequency range for pentacene. There may be other coupled modes outside our frequency window, but it is apparent that the modes that modulate the charge transport most strongly reside in the $\sim 1 \mathrm{THz}$ range. It is intuitive that the strongly coupled mode frequency is lower for crystalline organic semiconductors compared to polymeric ones: for crystalline organic semiconductors, conduction requires charge delocalization among different molecular moieties so that intermolecular modes will affect the conductivity most strongly. Intermolecular modes typically have a low frequency due to the weak intermolecular interactions. For semiconducting polymers, in contrast, one expects that intramolecular modes are responsible for modulating the conduction along the polymer backbone. These

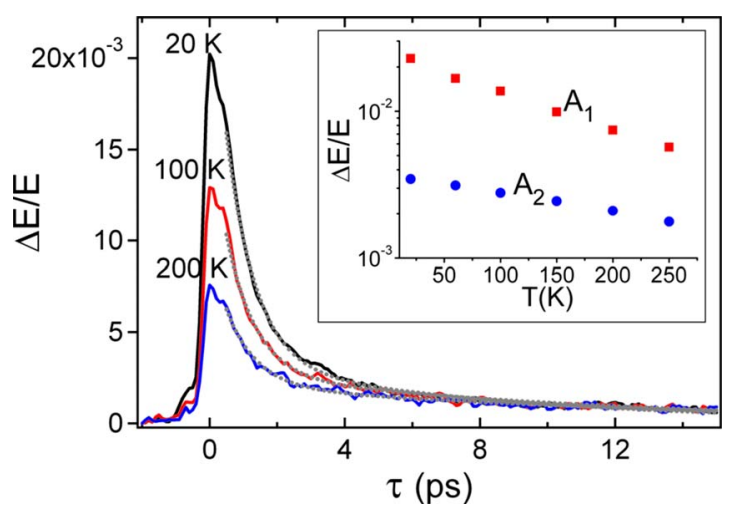

FIG. 5. (Color online) The spectrally integrated modulation $(\Delta E / E)$ as a function of delay time $\tau$ for pentacene when exciting with $400 \mathrm{~nm}$ pulses with a laser fluence of $20 \mathrm{~J} \mathrm{~m}^{-2}$. The traces are recorded at different temperatures: $20 \mathrm{~K}$ (black), $100 \mathrm{~K}$ (red), and $200 \mathrm{~K}$ (blue). For $\tau>0.5 \mathrm{ps,} \mathrm{each}$ modulation trace is fit by a function $\Delta E / E=A_{1} e^{-\tau / \tau_{1}}+A_{2} e^{-\tau / \tau_{2}}$ indicated by the gray dotted traces. The inset shows the parameters $A_{1}$ (the size of the CAT signal) and $A_{2}$ (the size of the free carrier contribution) as a function of temperature.

intramolecular modes typically have an appreciably higher frequency.

The amplitude of the $1.1 \mathrm{THz}$ resonance decays quickly $(\sim 1 \mathrm{ps})$ to a lower level, which we attribute to the thermalization of the carriers. In fact, the terahertz complex conductivity signal has two distinct contributions: from free carriers (mostly real and frequency independent, observed at long delay times ${ }^{7,20}$ ) and from carrier-activated terahertz (CAT) modes. We observe that at short times, the latter dominates the response; at long times free carriers dominate. This can be understood by noting that immediately after photoexcitation, carriers will be hot, and will relatively be delocalized. This will strengthen the CAT signal, which will diminish as carriers cool and become increasingly localized (as the CAT signals is approximately proportional to the fourth power of the localization length). In contrast, the free carrier signal is relatively insensitive to carrier cooling: the mobility has been shown to depend only very weakly on the precise carrier excited state. ${ }^{16}$ As a result, the CAT signal, superimposed on the free carrier signal, loses relative strength upon carrier thermalization. Additional evidence for these dynamics is given in Fig. 5, in which the spectrally integrated modulation is shown as a function of the delay time $\tau$ at various temperatures. The fit curves (indicated in Fig. 5 by the gray dotted lines) show that the plotted traces of the charge dynamics can be adequately described by a function of the form $\Delta E / E=A_{1} e^{-\tau / \tau_{1}}+A_{2} e^{-\tau / \tau_{2}}$ (whereas attempts to fit the data with a single exponential were unsuccessful). The fast component (predominantly due to the decay of the CAT signal) has a decay time $\tau_{1}$ of $0.8 \mathrm{ps} . \tau_{2}$, the decay time of the free carrier contribution, is temperature dependent (an observation that has been previously reported in Ref. 7) ranging from 8 to 14 ps between 20 and $200 \mathrm{~K}$.

The inset in Fig. 5 shows the amplitude of both the CAT $\left(A_{1}\right)$ and free carrier component $\left(A_{2}\right)$ as a function of temperature. Both components decrease with increasing temperatures, which is expected since (i) the CAT signal is strongly dependent on the hot carrier delocalization length (which decreases with increasing temperature ${ }^{17}$ ) and (ii) the 
free carrier signal at longer times is known to decrease with temperature. ${ }^{5-9}$ Note that the resonant CAT signature of the charge transport behavior is present for all delay times at both $20 \mathrm{~K}$ and room temperature (see Fig. 2), indicating that the conduction in the material is governed by one single mechanism at all temperatures. We would like to stress that the reduced intensity of the CAT signal at higher temperatures and later delay times is due to a reduction in the transition dipole moment of the relevant modes and not to a reduction in their importance in the dynamics of the charge. The CAT signal is present most strongly in the presence of hot charge carriers, but it has a finite amplitude even when the charge carriers have cooled, i.e., after several picoseconds.

In conclusion, terahertz transient conductivity measurements reveal that charge carriers in pentacene are strongly coupled to the low energy phonon modes that, in turn, modulate the probability of charge transfer between adjacent molecular moieties constituting the crystal. We expect such electron-phonon interactions to be a general characteristic of crystalline semiconductors.

This work is part of the research program of the Foundation for Fundamental Research on Matter (FOM) with financial support from The Netherlands Organization for the Advancement of Research (NWO). G.O. acknowledges the financial support PRIN No. 2005034397. A.T. thanks the Royal Society and the EPSRC-GB (U.K.). H.A.v.L. acknowledges the D.P.I. for their financial support.

${ }^{1}$ V. Podzorov, S. E. Sysoev, E. Loginova, V. M. Pudalov, and M. E. Gershenson, Appl. Phys. Lett. 83, 3504 (2003).

${ }^{2}$ C. D. Dimitrakopoulos, A. R. Brown, and A. Pomp, J. Appl. Phys. 80, 2501 (1996)

${ }^{3}$ H. Klauk, D. J. Gundlach, J. A. Nichols, and T. N. Jackson, IEEE Trans. Electron Devices 46, 1258 (1999).

${ }^{4}$ S. Yoo, B. Domercq, and B. Kippelen, Appl. Phys. Lett. 85, 5427 (2004).
${ }^{5}$ F. A. Hegmann, R. R. Tykwinski, K. P. H. Lui, J. E. Bullock, and J. E. Anthony, Phys. Rev. Lett. 89, 227403 (2002).

${ }^{6}$ O. Ostroverkhova, D. G. Cooke, S. Shcherbyna, R. F. Egerton, F. A. Heggman, R. R. Tykwinski, and J. E. Anthony, Phys. Rev. B 71, 035204 (2005).

${ }^{7}$ V. K. Thorsmølle, R. D. Averitt, X. Chi, D. L. Smith, A. P. Ramirez, and A. J. Taylor, Appl. Phys. Lett. 84, 891 (2004).

${ }^{8}$ O. D. Jurchescu, J. Baas, and T. T. M. Palstra, Appl. Phys. Lett. 84, 3061 (2004).

${ }^{9}$ W. Warta and N. Karl, Phys. Rev. B 32, 1172 (1985).

${ }^{10}$ Y. C. Cheng, J. Chen, L. N. Ding, T. H. Wong, P. D. Kleiber, and D. Lui, J. Chem. Phys. 118, 3764 (2003).

${ }^{11}$ N. Koch, I. Salzmann, J. Rabe, A. Vollmer, H. Weiss, and B. Nickel, Phys. Rev. Lett. 96, 156803 (2006).

${ }^{12}$ Z. Q. Li, V. Podzorov, N. Sai, A. Martin, M. E. Gershenson, M. Di Ventra, and D. N. Basov, Phys. Rev. Lett. 99, 016403 (2007).

${ }^{13}$ K. P. Pernstich, B. Rössner, and B. Battlog, Nat. Mater. 7, 321 (2008).

${ }^{14}$ K. Hannewald and P. A. Bobbert, Appl. Phys. Lett. 85, 1535 (2004).

${ }^{15}$ P. J. Brown, H. Sirringhaus, M. Harrison, M. Shkunov, and R. H. Friend, Phys. Rev. B 63, 125204 (2001).

${ }^{16}$ A. Troisi and G. Orlandi, Phys. Rev. Lett. 96, 086601 (2006).

${ }^{17}$ A. Troisi and G. Orlandi, J. Phys. Chem. A 110, 4065 (2006).

${ }^{18}$ M. C. Beard, G. M. Turner, and C. A. Schmuttenmaer, Phys. Rev. B 62, 15764 (2000)

${ }^{19}$ E. Hendry, J. M. Schins, L. P. Candeias, L. D. A. Siebbeles, and M. Bonn, Phys. Rev. Lett. 92, 196601 (2004).

${ }^{20}$ F. A. Hegmann, O. Ostroverkhova, and D. G. Cooke, in Photophysics of Molecular Materials: From Single Molecules to Single Crystals, edited by G. Lanzani (Wiley, New York, 2006), pp. 404-419.

${ }^{21}$ X. Ai, M. C. Beard, K. P. Knutsen, S. E. Shaheen, G. Rumbles, and R. J. Ellingson, J. Phys. Chem. B 110, 25462 (2006).

${ }^{22}$ J. Shan, F. Wang, E. Knoesel, M. Bonn, and T. F. Heinz, Phys. Rev. Lett. 90, 247401 (2003).

${ }^{23}$ O. D. Jurchescu, J. Baas, and T. T. M. Palstra, Appl. Phys. Lett. 87, 052102 (2005).

${ }^{24}$ E. Hendry, M. Koeberg, J. M. Schins, H. K. Nienhuys, V. Sundström, L. D. A. Siebbeles, and M. Bonn, Phys. Rev. B 71, 125201 (2005).

${ }^{25}$ M. Koeberg, E. Hendry, J. M. Schins, H. A. van Laarhoven, C. F. J. Flipse, K. Reimann, M. Woerner, T. Elsaesser, and M. Bonn, Phys. Rev. B 75, 195216 (2007).

${ }^{26}$ H. Nemec, F. Kadlec, and P. Kuzel, J. Chem. Phys. 117, 8454 (2002).

${ }^{27}$ S. Etemad, A. Pron, A. J. Heeger, A. G. MacDiarmid, E. J. Mele, and M. J. Rice, Phys. Rev. B 23, 5137 (1981).

${ }^{28}$ E. J. Mele and M. J. Rice, Phys. Rev. Lett. 45, 926 (1980).

${ }^{29}$ C. Castiglioni, J. T. L. Navarrete, G. Zerbi, and M. Gussoni, Solid State Commun. 65, 625 (1988). 